\title{
Cost-effectiveness of pertuzumab and trastuzumab as a first-line treatment of HER2-positive metastatic breast cancer in China
}

\author{
Hao Wang ${ }^{1 \#}$, Ye Wang ${ }^{2 \#}$, Ruixue Gong ${ }^{1}$, Yuyu Geng ${ }^{2}, \mathrm{Li} \mathrm{Li}^{1}$ \\ ${ }^{1}$ Department of Pharmacy, Nanjing Drum Tower Hospital, The Affiliated Hospital of Nanjing University Medical School, Nanjing, China; \\ ${ }^{2}$ Department of Clinical Pharmacy, China Pharmaceutical University, Nanjing, China \\ Contributions: (I) Conception and design: H Wang, L Li; (II) Administrative support: L Li; (III) Provision of study materials or patients: H Wang, Y \\ Wang; (IV) Collection and assembly of data: Y Geng, R Gong; (V) Data analysis and interpretation: H Wang, Y Wang, R Gong; (VI) Manuscript \\ writing: All authors; (VII) Final approval of manuscript: All authors. \\ \#These authors contributed equally to this work. \\ Correspondence to: Li Li. Department of Pharmacy, Nanjing Drum Tower Hospital, The Affiliated Hospital of Nanjing University Medical School, \\ Nanjing, China. Email: njgllily@163.com.
}

\begin{abstract}
Background: This study sought to evaluate the cost-effectiveness of a pertuzumab, trastuzumab, and docetaxel (PTD) regimen and a trastuzumab and docetaxel (TD) regimen in the first-line treatment of human epidermal growth factor receptor 2 (HER2)-positive metastatic breast cancer (MBC) in the context of the Chinese health system.

Methods: A 3 health-state Markov model was established to simulate the disease process of patients. Transition probability and adverse reactions data were derived from the CLEOPATRA trial. The utility value of the disease status was derived from the relevant literature, and the costs were based on the China Drug Database and other local charges. Sensitivity analyses were performed to assess the uncertainty of the results caused by parameter variability.

Results: Compared to the TD regimen, the PTD regimen afforded an additional 0.64 quality-adjusted lifeyears (QALYs) at an incremental cost of 44,828 USD. The incremental cost-effectiveness ratio (ICER) was 69,702 USD/QALY. The results of the sensitivity analysis suggest that the results are reliable.

Conclusions: The PTD regimen can prolong the life of patients and improve their quality of life with HER2-positive MBC, but the medical costs also increase accordingly. Based on the current payment threshold in China, the PTD regimen has no economic advantage over the TD regimen in the first-line treatment of HER2-positive MBC patients.
\end{abstract}

Keywords: Cost-effectiveness; pertuzumab; trastuzumab; Markov; breast cancer

Submitted Jul 09, 2021. Accepted for publication Oct 09, 2021.

doi: 10.21037/apm-21-2412

View this article at: https://dx.doi.org/10.21037/apm-21-2412

\section{Introduction}

According to the Global Cancer Incidence, Mortality and Prevalence (Globocan) data from the World Health Organization, there were approximately 2.26 million new breast cancer cases diagnosed, with breast cancer accounting for $11.7 \%$ of all new cancer diagnoses in women globally (1). In 2020, breast cancer surpassed lung cancer as the leading cause of cancer mortality among females worldwide. Breast cancer is one of the most common malignant tumors. In China, it ranks first in the incidence of malignant tumors in women and is considered the "first killer of women's health" (2). Cases in China account for 9.6\% of all breast cancer deaths worldwide (3). According to national cancer registrations there are 54.5 new breast cancer patients per 100,000 people in China, and of these, $6-7 \%$ of patients have a diagnosis of advanced breast cancer (4). After an initial diagnosis of early breast cancer, patients receive 
adjuvant therapy; however, $30 \%$ of patients eventually relapse or the cancer metastasizes (5). Thus, in the coming years, the proportion of advanced breast cancer in China will continue to increase, which will lead to a drastic increase in health expenditure, placing a burden on both society and patients' families.

About $15-30 \%$ of breast cancer patients suffer from human epidermal growth factor receptor 2 (HER2)-positive breast cancer (6). Due to its poor prognosis, HER2 breast cancer has been described as the "most dangerous breast cancer" (6). HER2 breast cancer has a higher malignancy of tumor cells, faster disease progression (PD), and is more prone to metastasis and recurrence than other types of breast cancer (6). With the advent of anti-HER2 breast cancer targeted drugs, trastuzumab has become the clinical gold standard for anti-HER2 breast cancer treatments. In the past 20 years, the treatment of HER2-positive breast cancer has made significant progress. Indeed, $3 / 4$ of early HER2-positive breast cancer patients achieve a clinical cure. However, among early HER2-positive breast cancer patients, $25 \%$ still experience disease recurrence after the current anti-HER2 treatment (7).

Pertuzumab is a recombinant humanized monoclonal antibody that targets the extracellular domain of the HER2 protein and prevents HER2 heterodimerization (8). According to the results of the international large-scale phase III clinical APHINITY trial, in comparison to the existing standard anti-HER2 treatment plan of trastuzumab combined chemotherapy, a combined chemotherapy regimen of pertuzumab plus trastuzumab can be used for postoperative adjuvant treatment and can reduce the risk of recurrence of HER2-positive early breast cancer disease or death by $19 \%$. Its efficacy is better than that of single-targeted drug therapy (9). Following the launch of pertuzumab on the Chinese mainland in 2019, clinical guidelines now recommend a first-line anti-HER-2 treatment of pertuzumab and trastuzumab combined with chemotherapy (10). This treatment may significantly reduce the risk of recurrence and death, open the era of dual-target therapy, and bring more hope of a cure to patients.

However, despite its significant clinical benefits, this treatment can also place a substantial economic burden on the patients and the healthcare system. In recent years, the CLEOPATRA study, which was a double-blind, randomized, placebo-controlled, phase 3 clinical study, sought to compare the efficacy and safety of pertuzumab, trastuzumab, and docetaxel (PTD), to placebo, trastuzumab, and docetaxel
(TD) in patients with HER2-positive metastatic breast cancer (MBC). Based on the CLEOPATRA 4-year follow-up trial (11), in 2013, Durkee et al. (12) constructed a Markov model to evaluate pertuzumab in the United States (US), and Diaby et al. (13-15) conducted a cost-effectiveness analysis of HER2 targeted therapy in different countries. Leung et al. (16) used Taiwan's national claims database to evaluate the economic aspects of HER2 first-line therapeutic drugs. These studies showed that pertuzumab and trastuzumab combined with chemotherapy as a first-line therapy was not cost-effective. The CLEOPATRA trial was updated on March 12, 2020, and the median progress-free survival time of patients in the PTD group was significantly longer than that of patients in the TD group (18.5 vs. 12.4 months; $\mathrm{P}<0.001$ ) (17). With 8 -year survival rates of $37 \%$ and $23 \%$, respectively, the PTD regimen had significantly more survival benefits than the TD regimen (17). Thus far, 2 pharmacoeconomic studies have been published based on the latest data from the CLEOPATRA trial. Moriwaki et al. (18) constructed the partitioned survival model from the Japanese public healthcare payers' perspective to evaluate the cost-effectiveness of HER2-positive MBC targeted therapy. The pertuzumab group gained 0.949 quality-adjusted life-years (QALYs) and had an incremental cost-effectiveness ratio (ICER) value of 183,901 USD/ QALY. In addition, Cheng et al. (19) also conducted an economic evaluation of PTD from the perspective of the medical system in Singapore and found that the pertuzumab group gained 0.73 more QALYs, and had an ICER value of 272,244 USD/QALY. The results in both countries show that pertuzumab combination therapy is unlikely to be a cost-effective treatment.

However, the conclusions reached by previous studies may not be applicable to China due to its different medical policies, background, medical service prices, and drug prices. To date, no economic comparison between these 2 HER2-positive MBC treatment regimens has been conducted in China; thus, it has not been determined which regimen is the most cost-effective. Based on the CLEOPATRA trial, we conducted a cost-effective analysis to evaluate the economic aspects of the PTD and TD regimens in the context of the Chinese health system. The results of this study will provide a reference that enables clinicians to select a better treatment plan in the treatment of HER2-positive MBC. We present the following article in accordance with the CHEERS reporting checklist (available at https://dx.doi.org/10.21037/apm-21-2412). 


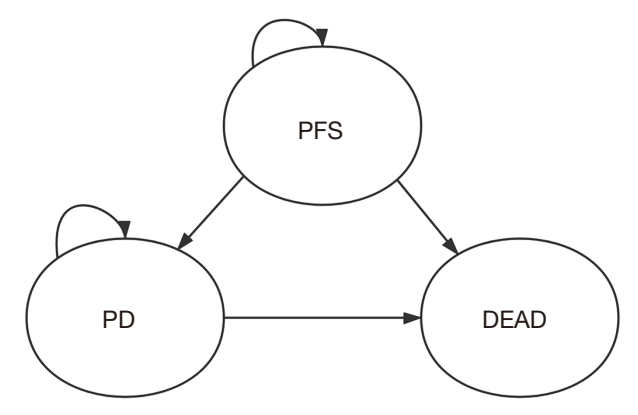

Figure 1 The 3-state heath model diagram. PFS, progression-free survival; $\mathrm{PD}$, progressive disease.

\section{Methods}

A Markov model was established using Treeage Pro Suit 2011 to evaluate the cost-effectiveness of PTD combination therapy in the Chinese health system. Only direct medical costs were calculated. The model included first-line treatment costs, adverse event management costs, subsequent therapy costs after PD, hospital service program costs, and follow-up costs. The incidence of adverse events was extracted from the CLEOPATRA trial (17), and the utility to the Chinese population was taken from previous research by Lloyd et al. (20). The US dollar is the standard international currency. Costs were calculated in local currency units (RMB) and converted into US dollars, increasing the comparability between different countries and regions of similar reports. All costs were converted into US dollars according to the Chinese Consumer Price Index in 2021 ( 1 USD =6.7518 Chinese RMB). A $5 \%$ annual discount rate was applied to the long-term costs and health utilities.

The results of the model are expressed using the following terms: QALYs, cumulative costs (COSTs), and ICER, the latter being commonly applied costeffectiveness assessments, where it is synonymous with the COST per QALY gained (21). The cost-effectiveness of the intervention program is determined by the ICER and willingness to pay (WTP). Based on the China Guidelines for Pharmacoeconomic Evaluations (22), an amount 3 times the per capita gross domestic product (GDP; USD 31,511) in 2020 was taken as the willingness-to-pay threshold. In addition, a deterministic sensitivity analysis (DSA) and a probabilistic sensitivity analysis (PSA) were performed to investigate the stability of the model. As the economic analysis was based on a literature review and experimental models, the approval of an institutional review board or ethics committee was not required.

\section{Model structure}

The Markov model plays an important role in estimating the value of cancer treatments (23). Modelling in costeffectiveness provides a common framework by which to systematically assess costs and outcomes, represent uncertainty, and compare alternative courses of action (21). The decision-making model comprised 3 key health states: (I) the progression-free survival (PFS) state (the initial state); (II) the PD state; and (III) the death state (terminal state) (see Figure 1). It was assumed that over time, patients gradually progress from PFS to PD and from PD to death. The cycle length of the Markov model was 21 days, which was consistent with the trial. Following advice from local clinicians, the model simulation time was 20 years.

\section{Clinical data}

The study was conducted in accordance with the Declaration of Helsinki (as revised in 2013). According to the CLEOPATRA trial (17), patients with HER2 positive MBC were randomly assigned to the PTD group or the TD group. All patients were assumed to be in the PFS state and received one of the following treatments at the beginning of the model: (I) PTD: pertuzumab was administered on day 1 of each (3-week) cycle, starting with a loading dose of $840 \mathrm{mg}$ in the first cycle, followed by a dose of $420 \mathrm{mg}$ in the subsequent cycles. Trastuzumab was administered on day 2 , and the loading dose of the first cycle was 8 and $6 \mathrm{mg} / \mathrm{kg}$ in subsequent cycles. Docetaxel was administered on day 2 of each cycle at $75 \mathrm{mg} / \mathrm{m}^{2}$ per cycle, increasing to $100 \mathrm{mg} / \mathrm{m}^{2}$ if tolerated for at least 6 cycles; and (II) TD: the method of administration was the same as that of the PTD group until PD or death; however, pertuzumab was replaced with a placebo of the same appearance and quality.

The median follow-up period was 99.9 months [interquartile range (IQR) 92.9-106.4] in the PTD group and 98.7 months (90.9-105.7) in the TD group. The median PFS of patients was 18.7 versus 12.4 months [PTD vs. TD; hazard ratio (HR): 0.69, 95\% confidence interval (CI): 0.590.81 ], and the median overall survival (OS) was 57.1 months and 40.8 months (HR: 0.69, 95\% CI: 0.58-0.82).

\section{Statistical analysis and transition probability}

Engauge Digitizer version was used to extract the KaplanMeier (KM) curves for PFS and OS from the CLEOPATRA trial. Following the approach of Guyot et al. (24), 
Table 1 Results of the fit to the observed data

\begin{tabular}{|c|c|c|c|c|c|c|}
\hline Variables & Weibull & Exponential & Log-logistic & Log-normal & Gompertz & Gen gamma \\
\hline $\mathrm{AIC}$ & $3,009.204$ & $3,011.922$ & $2,947.52$ & 2,949.092 & $2,970.58$ & $2,945.94$ \\
\hline $\mathrm{BIC}$ & $3,017.197$ & $3,015.918$ & $2,955.513$ & $2,957.085$ & $2,978.573$ & 2,957.929 \\
\hline \multicolumn{7}{|l|}{ PTD OS } \\
\hline $\mathrm{BIC}$ & $2,815.912$ & $2,819.949$ & $2,797.132$ & $2,790.61$ & $2,815.621$ & $2,795.361$ \\
\hline \multicolumn{7}{|l|}{ TD PFS } \\
\hline $\mathrm{AIC}$ & $3,024.303$ & $3,024.951$ & $2,919.281$ & $2,925.49$ & $2,973.779$ & $2,904.733$ \\
\hline $\mathrm{BIC}$ & $3,032.316$ & $3,028.957$ & 2,927.294 & $2,933.501$ & $2,981.792$ & $2,916.753$ \\
\hline $\mathrm{BIC}$ & $3,112.666$ & $3,115.799$ & $3,090.361$ & $3,102.647$ & $3,121.724$ & $3,103.708$ \\
\hline
\end{tabular}

PTD, pertuzumab, trastuzumab and docetaxel arm; PFS, progression-free survival; AIC, Akaike information criterion; BIC, Bayesian information criterion; OS, overall survival; TD, trastuzumab and docetaxel.

Table 2 Best fit and the values of the parameters

\begin{tabular}{lccc}
\hline Variables & Best fitting & Parameters $(\mu / \lambda)$ & Parameters $(\sigma / \gamma)$ \\
\hline PTD PFS & Gen-gamma & $\mu=3.256$ & $\sigma=1.261$ \\
PTD OS & Log-normal & $\mu=4.492$ & $\sigma=1.102$ \\
TD PFS & Gen-gamma & $\mu=2.719$ & $\sigma=1.0318$ \\
TD OS & Log-logistic & $\lambda=4.482$ & $\gamma=0.645$ \\
\hline
\end{tabular}

PTD, pertuzumab, trastuzumab and docetaxel arm; PFS, progression-free survival; OS, overall survival; TD, trastuzumab and docetaxel.

the Survival Analysis in Health Economic Evaluation (survHE) package in $\mathrm{R}$ language (4.0.4) was used to reconstruct the patient-level data and fit the KM curves. Exponentia, Weibull, log-logistic, log-normal, gompertz, and gen-gamma distribution functions were considered to extrapolate the probability of survival to cover the lifetime horizon. The optimal function distribution was judged by a visual inspection, a comparison of the median PFS and the median OS, the Akaike information criterion (AIC), and the Bayesian information criterion (BIC). Finally, gengamma was selected to fit the PFS curve of the PTD and TD groups. Log-normal and log-logistic distributions were found to fit the OS curve of PTD and TD groups, respectively. The 2 optimal fitting distributions and related parameters are shown in Tables 1 and 2, and the original curve and the fitting curve are shown in Figure 2.

Determining time-dependent transition probability is a key step in the 3-state Markov model for pharmacoeconomic evaluation. Black et al. (25) introduced the method of determining the transition probability of the Markov model. The TD group was taken as an example. In the previous section, the PFS distribution parameters obtained from the KM curve were substituted into the gengamma distribution model. We can calculate the transition probability from the PFS state to the next progressionfree state (pFTF). We assumed that pFTD was equal to the natural mortality rate of $7.04 \%$ issued by the Chinese Bureau of Health Statistics in 2020. As the DEALA principle states, pFTP was 1-pFTF-pFTD. Similarly, by substituting OS distribution parameters into the log-logistic distribution model, the probability of transition from survival state to the next survival state (pSTS) and pSTD can be obtained. According to Zhou et al. (26), the corrected pPTP is derived from pSTS (including 3 parts of pFTF, 

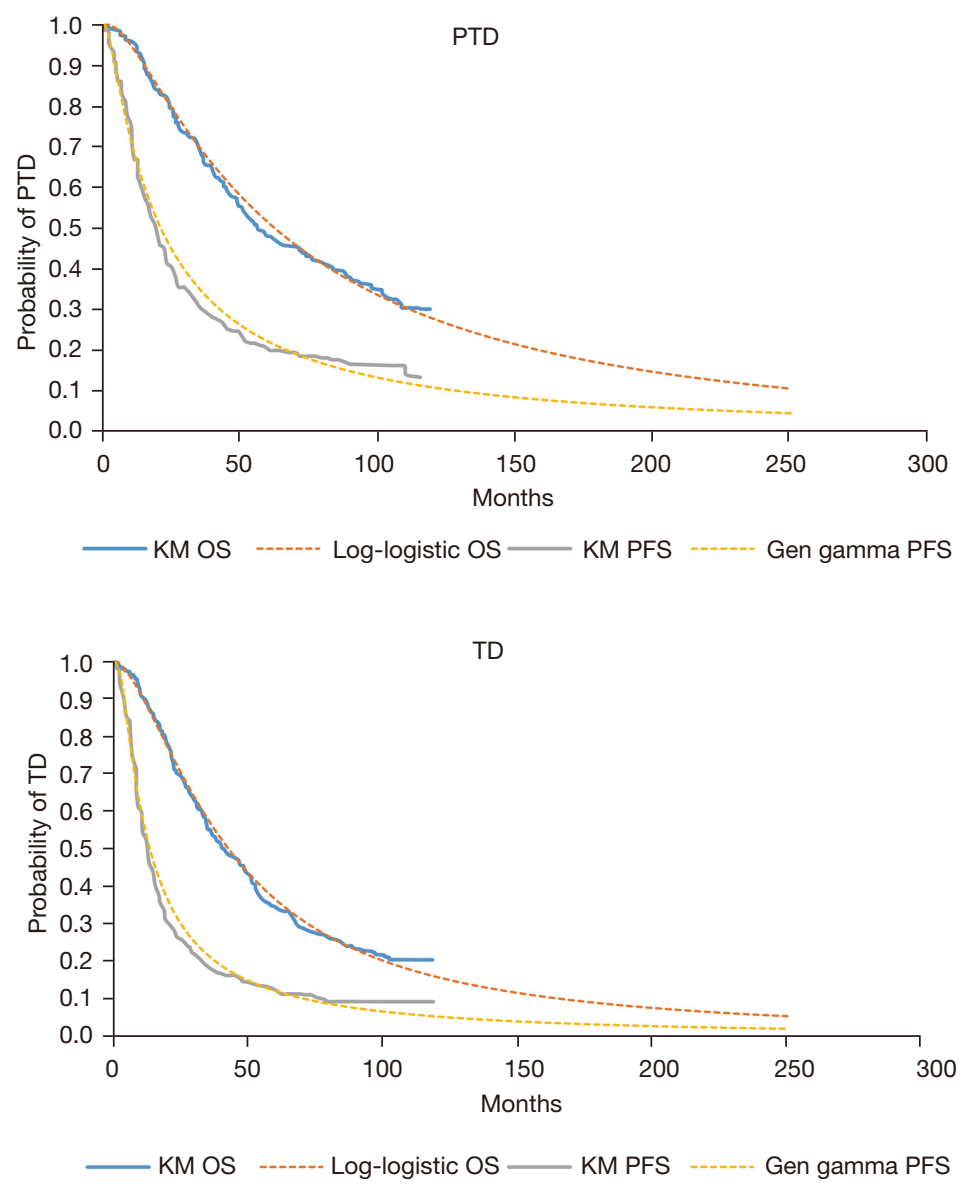

Figure 2 Results of the survival curve as fit to the PTD arm and TD arm. PTD, pertuzumab, trastuzumab and docetaxel arm; TD, trastuzumab and docetaxel; OS, overall survival; PFS, progression-free survival; KM, Kaplan-Meier.

pFTD, and $\mathrm{pPTP})$, then the transition probability from PD state to DEAD state is $\mathrm{nPTD}=1-\mathrm{pPTP}$. The formula is:

$$
n P T P=\frac{[(n P F S+n P D) \times p S T S-n P F S \times p F T F-n P F S \times p F T D]}{n P D}
$$

where pFTF is the transition probability from the PFS state to the next PFS state; pFTD is the transition probability from the PFS state to the DEAD state; pFTP is the transition probability from the PFS state to the PD state; pSTS is the transition probability from the survival state to the next survival state; pSTD is the transition probability from the survival state to the DEAD state; pPTP is the transition probability from the PD state to the next PD state; pPTD is the transition probability from the $\mathrm{PD}$ state to the DEAD state; nPFS is patients in the PFS state in the last cycle; and $\mathrm{nPD}$ is patients in the PD state in the last cycle.

\section{Medical costs}

The context of the healthcare system in China was adopted to estimate the direct medical costs, including drug treatment costs, adverse event treatment costs, follow-up costs, hospital service costs, and subsequent therapy costs, using a 21-day cycle. The treatment costs were calculated using the drug delivery schedule and the unit costs of the drugs, and the prices were based on the median prices of the bid-winning products as per the winning price on the China Medical Information Network (https://www.menet.com. $\mathrm{cn})$. The prices of pertuzumab, trastuzumab, trastuzumab emtansine, and lapatinib from Roche are relatively fixed. The prices of other drugs were based on the average bidwinning prices of drugs in various provinces of China. The drug doses were calculated for a standard human body surface area of $1.72 \mathrm{~m}^{2}$ and a standard female bodyweight 
of $55 \mathrm{~kg}$; the waste of drugs was not considered in the calculation process. Pertuzumab, trastuzumab, trastuzumab emtansine, docetaxel, methylprednisolone sodium succinate, and cyclophosphamide were administered intravenously, and the configuration fee and intravenous infusion fee for the chemotherapeutic drugs were calculated. Other drugs were administered orally and did not have auxiliary administration costs.

The follow-up costs and medical service costs mainly included diagnosis fees, nursing fees, hospitalization fees, intravenous infusion fees, management costs, electrocardiogram costs, routine blood costs, biochemistry costs, blood coagulation costs, tumor marker costs, sex hormone costs, enhanced computed tomography costs, and bone scanning costs. The costs were based on the average price of medical services in different provinces of China. The costs of adverse events of grade 3 or 4 , including leukopenia, neutropenia, diarrhea, fatigue, asthenia, nausea, vomiting, constipation, headache, neuropathy peripheral, and anemia, were considered. The incidence and treatment costs of adverse events were retrieved from the relevant literature, and selection was based on the opinions of clinical experts. Subsequent therapy and its proportion were derived from the supplementary data of the CLEOPATRA trial, and the post-progressive treatment costs of the 2 groups were calculated according to the proportion.

\section{Utility value}

To date, no study on the quality of life of HER2 positive MBC patients has been conducted in China; thus, the utility value used in this analysis was obtained from Lloyd's study (20). The utility values estimated for the PFS and PD states were 0.715 and 0.443 , respectively. After calculating the disutilities of adverse events, such as neutropenia, leukopenia, and diarrhea, the total adverse event utility values of the 2 groups were -0.129 and -0.116 , respectively (these values were mainly based on the study of Nafees and Lloyd) (20,27). All key cost inputs for the cost-utility model are shown in Table 3.

\section{Sensitivity analysis}

A one-way sensitivity analysis and a PSA of the model's parameters were performed to explore the uncertainty of the evaluation model. The distribution type and value range of the related parameters are shown in Table 3. In the oneway sensitivity analysis, the range of cost parameters was obtained from the China Medical Information Database and the standards of medical care. Any parameters for which a range of values could not be obtained were floated by $20 \%$ based on the base value. In the PSA, we set the cost parameter as the gamma distribution and the utility value as the beta distribution, extracting the values of the corresponding distribution for 10,000 Monte Carlo simulations. The results of the PSA are represented by an incremental cost-effectiveness scatter plot and acceptable curve. The actual processes occur in continuous time, with transitions and other events occurring throughout the period. However, the Markov process is a discrete snapshot sequence, and the expected value overestimates the life expectancy by about half a cycle (28). A half-cycle correction can be adopted to "correct" for overestimation.

\section{Results}

\section{Base-case analysis}

The base-case analysis of the 2 treatment regimens (see Table 4) showed that the QALYs of the PTD and TD groups were 2.28 and 2.92, respectively. Pertuzumab conferred 0.64 additional QALYs, but the corresponding cost was 44,828 USD. The ICER of PTD therapy compared to TD therapy was estimated to be 69,702 USD/QALYs. The results indicated that TD therapy was more cost-effective than PTD therapy as a first-line treatment for HER2-positive MBC.

\section{Sensitivity analysis}

The results of the one-way sensitivity analysis are shown in Figure 3. The utility value in the PFS stage and pertuzumab price were the most important factors affecting the Markov model. The discount rate, the utility value in the PD stage, and trastuzumab price also affected the analysis results; however, other parameters had little effect on the ICER value. In the sensitivity analysis, ICER values varied from 59,091 USD/QALY to 84,957 USD/QALY.

\section{$P S A$}

A PSA was performed to measure the effect of overall uncertainty on the model results, which required parameter distributions to be integrated into the model. The incremental cost-effectiveness scatter diagram, and the costeffectiveness acceptance curve of the total population were obtained through Monte Carlo simulation of 10,000 times (see Figures 4 and 5). All the scatter points were distributed 
Table 3 Summary of main medical costs, utility values, and other parameters

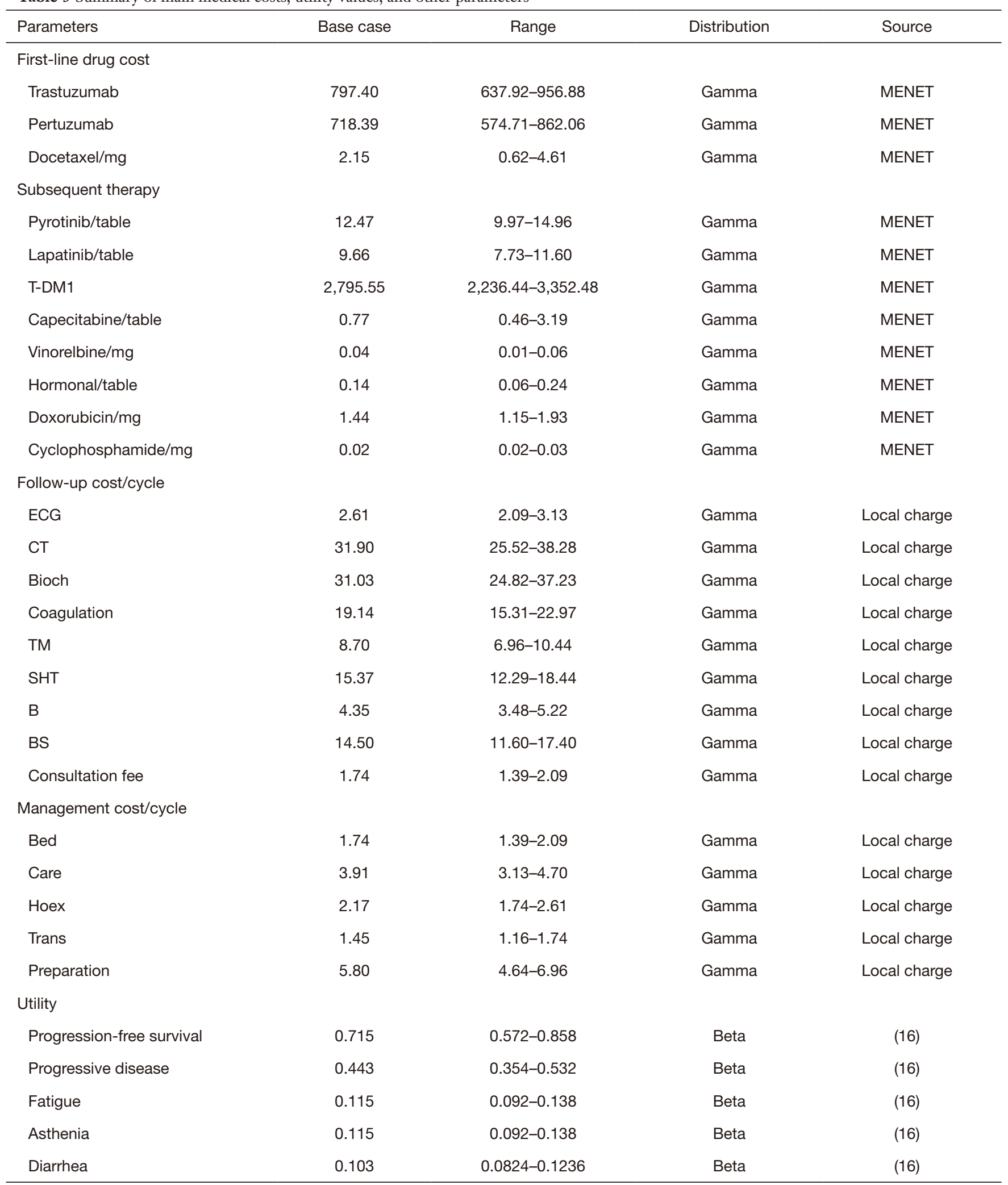

Table 3 (continued) 
Table 3 (continued)

\begin{tabular}{|c|c|c|c|c|}
\hline Parameters & Base case & Range & Distribution & Source \\
\hline Vomiting & 0.103 & $0.082-0.124$ & Beta & (16) \\
\hline Headache & 0.12 & $0.096-0.144$ & Beta & (21) \\
\hline Anemia & 0.073 & $0.058-0.088$ & Beta & (21) \\
\hline Hypertension & 0.044 & $0.035-0.053$ & Beta & (21) \\
\hline \multicolumn{5}{|l|}{ Others } \\
\hline Discount rate & $5 \%$ & $0-8 \%$ & Fixed in PSA & \\
\hline
\end{tabular}

T-DM1, trastuzumab emtansine; ECG, electrocardiograph; CT, computed tomography; TM, tumor markers; SHT, sex hormones; B, B-ultrasonography; BS, bone scanning; Hoex, hospitalization examination; PSA, probabilistic sensitivity analysis.

Table 4 Results of the base-case analysis

\begin{tabular}{lccccc}
\hline Variables & QALY & Incremental QALY & Cost (USD) & Incremental cost & ICER (USD/QALY) \\
\hline TD & 2.28 & - & 107,289 & - & - \\
PTD & 2.92 & 0.64 & 152,117 & 44,828 & 69,702 \\
\hline
\end{tabular}

QALY, quality-adjusted life-year; ICER, incremental cost-effectiveness ratio; TD, trastuzumab and docetaxel; PTD, pertuzumab, trastuzumab and docetaxel arm.

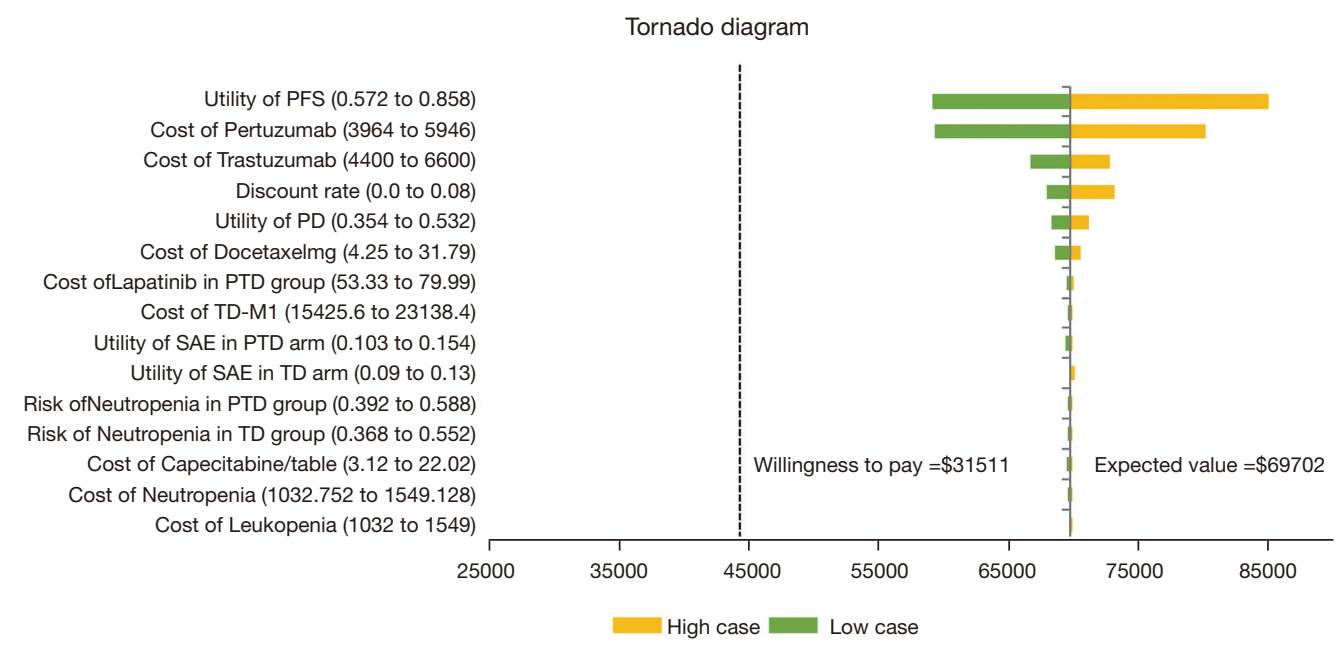

Figure 3 Tornado diagram of the one-way sensitivity analysis. PFS, progression-free survival; PD, disease progression; PTD, pertuzumab, trastuzumab and docetaxel arm; SAE, serious adverse event.

above the threshold of the WTP. The cost-utility acceptability curve showed that when the WTP was set to $\$ 90,000$, PTD had a cost-utility of $100 \%$ compared to
TD. The results of the PSA indicate that the pertuzumab treatment regimen had no economic advantage over the traditional trastuzumab treatment regimen in China. 


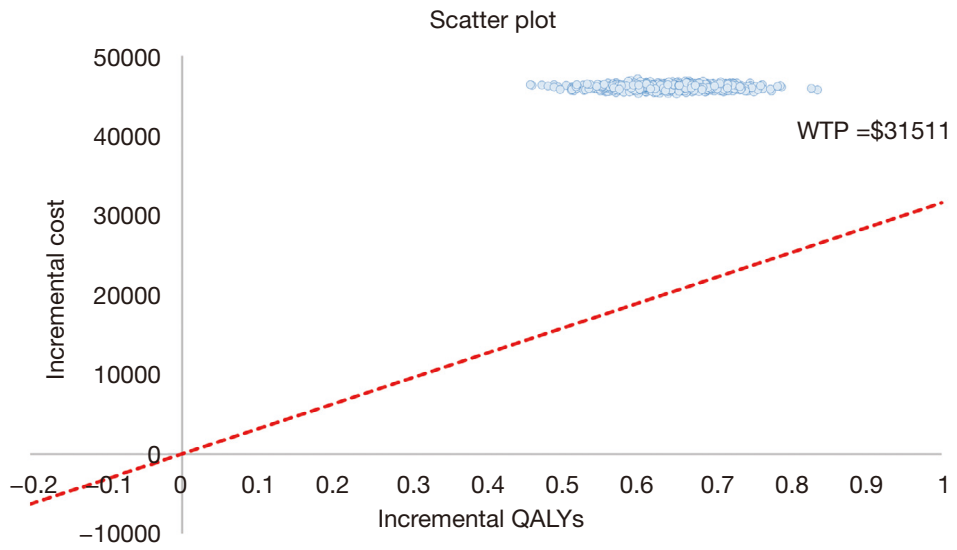

Figure 4 Scatter plot of incremental cost-effectiveness ratio in the Markov model. QALY, quality-adjusted life-year; WTP, willingness to pay.

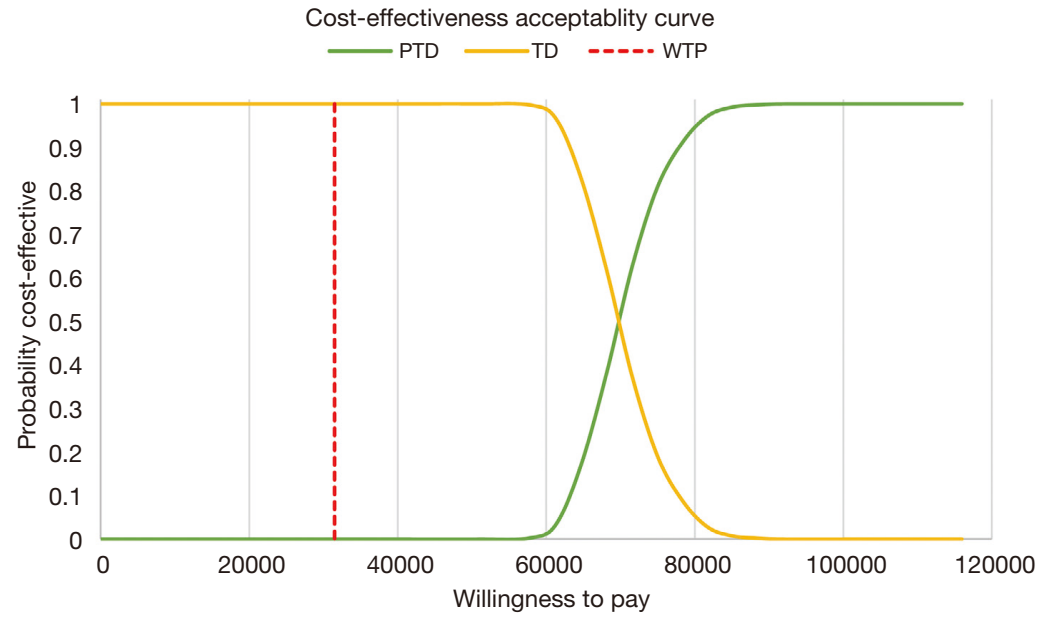

Figure 5 Cost-effectiveness acceptability curve in the Markov model. PTD, pertuzumab, trastuzumab and docetaxel arm; TD, trastuzumab and docetaxel; WTP, willingness to pay.

\section{Discussion}

Pertuzumab was initially approved in the US in 2012, and the dual-target therapy in combination with TD has become the international standard for the first-line treatment of HER2-positive MBC. In December 2018, pertuzumab received marketing authorization in China. In 2019, the Chinese Society of Clinical Oncology (CSCO) incorporated the PTD regimen into their guidelines for the diagnosis and treatment of breast cancer (10), recommending it as the first-line treatment for patients with HER2-positive $\mathrm{MBC}$ or recurrent breast cancer. Due to its short time on the market, no economic evaluation has been undertaken of the pertuzumab regimen in China. Although the clinical efficacy of the pertuzumab is widely recognized, the high cost of dual-target therapy is still an obstacle, and it is unlikely to be a cost-effective treatment. With the maturity of the CLEOPATRA trial and the incorporation of dualtarget drugs into the national health insurance scheme, the cost of pertuzumab decreases, and the clinical benefits are significant. However, the cost-effectiveness of dual-target therapy is still unknown, and further exploration is needed to provide guidance to clinicians and support medical insurance decisions in China.

The CLEOPATRA trial was updated in 2020 with a mean follow-up period of 8 years. Thus far, 2 pharmacoeconomic studies have been published based on the latest data of the CLEOPATRA trial. Moriwaki et al. (18) constructed the partitioned survival model from the Japanese public healthcare payers' perspective to evaluate 
the cost-effectiveness of HER2-positive MBC targeted therapy. The pertuzumab group gained 0.949 QALYs and had an ICER value of 183,901 USD/QALY. In addition, Cheng et al. (19) also conducted an economic evaluation of PTD from the perspective of the medical system in Singapore and found that the pertuzumab group gained 0.73 more QALYs, and had an ICER value of 272,244 USD/QALY. The results in both countries show that pertuzumab combination therapy is unlikely to be a costeffective treatment. The present study also reached a similar conclusion. Specifically, we found that the PTD group had greater budgetary challenges than the TD group, despite achieving more QALYs. The results of the basic analysis and sensitivity analysis showed that the ICER of PTD compared to that of TD reached 69,702 USD/QALYs, which was more than 3 times the per capita GDP, indicating that it provides no economic advantage.

To incorporate valuable new drugs into the drug list and achieve drug accessibility in a timely manner, the Chinese government has recently been conducting drug access negotiations and adjusting the drug list. In 2019, the latest round of national medical insurance negotiations resulted in the inclusion of 97 drugs on the list of B drugs, including pertuzumab. After the negotiations, drug prices were cut by an average of $60.7 \%$, which reduced the burden placed on patients by more than $80 \%$ and, in the case of some drugs, by more than $95 \%$. The present study used trastuzumab costs based on price negotiation data. However, according to our research results, pertuzumab is still overpriced in China. Conversely, given the uneven level of economic development between China's regions, in 2020, the highest per capita GDP province was nearly 5 times that of the lowest per capita GDP province. If the WTP threshold is set separately based on the GDP of each province in 2020, PTD will have economic advantages over TD in Beijing and Shanghai.

In August 2020, a trastuzumab biosimilar (Zercepac) was launched and has since been evaluated as an originator reference product. In a clinical trial, Zercepac demonstrated equivalent efficacy and similar safety and immunogenicity to trastuzumab among patients with HER2-positive recurrent breast cancer or MBC $(29,30)$. As the price of Hanquyou is lower than that of the original research drug, the economic aspects of the PTD scheme can be further improved.

The economic aspects of the pertuzumab regimen in China will gradually improve due to socio-economic development, an increase in the per capita GDP, the decline in drug prices driven by national health insurance policies, and competition from generic drugs. Although life is priceless, medical resources are limited or scarce, and all medical sectors and decision-makers have been restricted by budgets (21). Therefore, we need to conduct an economic evaluation of the PTD regimen and aim to provide reasonable information to help China's medical insurance policy decisions. In addition, foreign studies have shown that the time that patients spend at the end-of-life stage is often more valuable to them and to society than the same amount of time spent at any other stage of life (31). Britain and the US have introduced policies that target treatment at the end-of-life stage and that have increased the threshold amount that the average society is willing to pay. Thus, when combined with China's economic development level and the average WTP, exploring and perfecting the reasonable price and medical insurance payment standard of pertuzumab will further reduce the price of pertuzumab and promote the popularization of dual-target therapy in the treatment of HER2-positive breast cancer in China, prolonging the survival time of patients, and improving their quality of life.

This study had some limitations. First, the utility value referenced in this study was based on a UK patient population, as there is no relevant research on the measurement of utility value in Chinese HER2positive MBC patients at present; thus, specific research needs to be conducted on the utility value for Chinese patients. Additionally, the utility value of PFS had the most significant effect on the ICER value in the sensitivity analysis; thus, health output might affect the research results to a certain extent. Second, the adverse events in this study involving HER2-positive MBC patients were only assumed to be handled in the first cycle; however, there are many types of adverse events in reality, and their incidence generally varies with different disease states of patients or changes over time. Consequently, the data on the processing costs of adverse events may be underestimated.

\section{Conclusions}

According to the results of the basic analysis and sensitivity analysis, the dual-target therapy of pertuzumab might not have an economic advantage over trastuzumab at a WTP threshold of 3 times the per capita GDP in the context of the Chinese healthcare system. These findings can be used to provide guidance to clinicians and inform government policy in China. 


\section{Acknowledgments}

Funding: None.

\section{Footnote}

Reporting Checklist: The authors have completed the CHEERS reporting checklist. Available at https://dx.doi. org/10.21037/apm-21-2412

Data Sharing Statement: Available at https://dx.doi. org/10.21037/apm-21-2412

Conflicts of Interest: All authors have completed the ICMJE uniform disclosure form (available at https://dx.doi. org/10.21037/apm-21-2412). The authors have no conflicts of interest to declare.

Ethical Statement: The authors are accountable for all aspects of the work in ensuring that questions related to the accuracy or integrity of any part of the work are appropriately investigated and resolved. The study was conducted in accordance with the Declaration of Helsinki (as revised in 2013).

Open Access Statement: This is an Open Access article distributed in accordance with the Creative Commons Attribution-NonCommercial-NoDerivs 4.0 International License (CC BY-NC-ND 4.0), which permits the noncommercial replication and distribution of the article with the strict proviso that no changes or edits are made and the original work is properly cited (including links to both the formal publication through the relevant DOI and the license). See: https://creativecommons.org/licenses/by-nc-nd/4.0/.

\section{References}

1. Sung H, Ferlay J, Siegel RL, et al. Global Cancer Statistics 2020: GLOBOCAN Estimates of Incidence and Mortality Worldwide for 36 Cancers in 185 Countries. CA Cancer J Clin 2021;71:209-49.

2. Anastasiadi Z, Lianos GD, Ignatiadou E, et al. Breast cancer in young women: an overview. Updates Surg 2017;69:313-7.

3. Fan L, Strasser-Weippl K, Li JJ, et al. Breast cancer in China. Lancet Oncol 2014;15:e279-89.

4. Khodari W, Sedrati A, Naisse I, et al. Impact of locoregional treatment on metastatic breast cancer outcome: a review. Crit Rev Oncol Hematol 2013;87:69-79.

5. Soh J, Komoike Y, Mitsudomi T. Surgical therapy for pulmonary metastasis of breast cancer. Transl Cancer Res 2020;9:5044-52.

6. Yamashita S, Hattori N, Fujii S, et al. Multi-omics analyses identify HSD17B4 methylation-silencing as a predictive and response marker of HER2-positive breast cancer to HER2-directed therapy. Sci Rep 2020;10:15530.

7. Perez EA, Romond EH, Suman VJ, et al. Trastuzumab plus adjuvant chemotherapy for human epidermal growth factor receptor 2-positive breast cancer: planned joint analysis of overall survival from NSABP B-31 and NCCTG N9831. J Clin Oncol 2014;32:3744-52.

8. Adams CW, Allison DE, Flagella K, et al. Humanization of a recombinant monoclonal antibody to produce a therapeutic HER dimerization inhibitor, pertuzumab. Cancer Immunol Immunother 2006;55:717-27.

9. von Minckwitz G, Procter M, de Azambuja E, et al. Adjuvant Pertuzumab and Trastuzumab in Early HER2Positive Breast Cancer. N Engl J Med 2017;377:122-131. Erratum in: N Engl J Med 2017;377:702. Erratum in: N Engl J Med 2018;379:1585.

10. Breast Cancer Expert Committee of National Cancer Quality Control Center; Breast Cancer Expert Committee of China Anti-Cancer Association; Cancer Drug Clinical Research Committee of China Anti-Cancer Association. Guidelines for clinical diagnosis and treatment of advanced breast cancer in China (2020 Edition). Zhonghua Zhong Liu Za Zhi 2020;42:781-97.

11. Swain SM, Kim SB, Cortés J, et al. Pertuzumab, trastuzumab, and docetaxel for HER2-positive metastatic breast cancer (CLEOPATRA study): overall survival results from a randomised, double-blind, placebo-controlled, phase 3 study. Lancet Oncol 2013;14:461-71.

12. Durkee BY, Qian Y, Pollom EL, et al. Cost-Effectiveness of Pertuzumab in Human Epidermal Growth Factor Receptor 2-Positive Metastatic Breast Cancer. J Clin Oncol 2016;34:902-9.

13. Diaby V, Adunlin G, Ali AA, et al. Cost-effectiveness analysis of 1 st through 3 rd line sequential targeted therapy in HER2-positive metastatic breast cancer in the United States. Breast Cancer Res Treat 2016;160:187-96.

14. Diaby V, Ali AA, Williams KJ, et al. Economic evaluation of sequencing strategies in HER2-positive metastatic breast cancer in Mexico: a contrast between public and private payer perspectives. Breast Cancer Res Treat 2017;166:951-63.

15. Diaby V, Alqhtani H, van Boemmel-Wegmann S, et al. 
A cost-effectiveness analysis of trastuzumab-containing treatment sequences for HER-2 positive metastatic breast cancer patients in Taiwan. Breast 2020;49:141-8.

16. Leung HWC, Chan ALF, Muo CH, et al. Costeffectiveness of pertuzumab combined with trastuzumab and docetaxel as a first-line treatment for HER-2 positive metastatic breast cancer. Expert Rev Pharmacoecon Outcomes Res 2018;18:207-13.

17. Swain SM, Miles D, Kim SB, et al. Pertuzumab, trastuzumab, and docetaxel for HER2-positive metastatic breast cancer (CLEOPATRA): end-of-study results from a double-blind, randomised, placebo-controlled, phase 3 study. Lancet Oncol 2020;21:519-30.

18. Moriwaki K, Uechi S, Fujiwara T, et al. Economic Evaluation of First-Line Pertuzumab Therapy in Patients with HER2-Positive Metastatic Breast Cancer in Japan. Pharmacoecon Open 2021. doi: 10.1007/s41669-02000254-3. [Epub ahead of print].

19. Cheng LJ, Loke L, Lim EH, et al. Cost-effectiveness of pertuzumab and trastuzumab biosimilar combination therapy as initial treatment for HER2-positive metastatic breast cancer in Singapore. Expert Rev Pharmacoecon Outcomes Res 2021;21:449-56.

20. Lloyd A, Nafees B, Narewska J, et al. Health state utilities for metastatic breast cancer. Br J Cancer 2006;95:683-90.

21. Edlin R, McCabe C, Hulme C, et al. Cost-effectiveness modelling for health technology assessment: a practical course. Springer, 2015.

22. Liu G, Wu J, Sun LJB, China: Chinese Pharmaceutical Association. China guidelines for pharmacoeconomic evaluations; 2019

23. Bullement A, Cranmer HL, Shields GE. A Review of Recent Decision-Analytic Models Used to Evaluate the

Cite this article as: Wang H, Wang Y, Gong R, Geng Y, Li L. Cost-effectiveness of pertuzumab and trastuzumab as a first-line treatment of HER2-positive metastatic breast cancer in China. Ann Palliat Med 2021;10(11):11382-11393. doi: 10.21037/apm-212412
Economic Value of Cancer Treatments. Appl Health Econ Health Policy 2019;17:771-80.

24. Guyot P, Ades AE, Ouwens MJ, et al. Enhanced secondary analysis of survival data: reconstructing the data from published Kaplan-Meier survival curves. BMC Med Res Methodol 2012;12:9.

25. Black WC, Nease RF Jr, Welch HG. Determining transition probabilities from mortality rates and autopsy findings. Med Decis Making 1997;17:87-93.

26. Zhou T, Ma AX, Fu LY. Discusion on the Calculation of Markov Model Transition Probability in Pharmacoeconomics Evaluation. Chinese Health Economics 2017;36:40-2.

27. Nafees B, Lloyd AJ, Dewilde S, et al. Health state utilities in non-small cell lung cancer: An international study. Asia Pac J Clin Oncol 2017;13:e195-203.

28. Sonnenberg FA, Beck JR. Markov models in medical decision making: a practical guide. Med Decis Making 1993;13:322-38.

29. Xie L, Zhang E, Xu Y, et al. Demonstrating Analytical Similarity of Trastuzumab Biosimilar HLX02 to Herceptin ${ }^{\circledR}$ with a Panel of Sensitive and Orthogonal Methods Including a Novel Fc $\gamma$ RIIIa Affinity Chromatography Technology. BioDrugs 2020;34:363-79.

30. Xu B, Zhang Q, Sun T, et al. Efficacy, Safety, and Immunogenicity of HLX02 Compared with Reference Trastuzumab in Patients with Recurrent or Metastatic HER2-Positive Breast Cancer: A Randomized Phase III Equivalence Trial. BioDrugs 2021;35:337-50.

31. Round J. Is a QALY still a QALY at the end of life? J Health Econ 2012;31:521-7.

(English Language Editor: L. Huleatt) 